INT-PUB 05-06

\title{
Effective Lagrangian and Topological Interactions in Supersolids
}

\author{
D. T. Son \\ Institute for Nuclear Theory, University of Washington, Seattle, Washington 98195-1550
}

(Dated: January 2005)

\begin{abstract}
We construct a low-energy effective Lagrangian describing zero-temperature supersolids. Galilean invariance imposes strict constraints on the form of the effective Lagrangian. We identify a topological term in the Lagrangian that couples superfluid and crystalline modes. For small superfluid fractions this interaction term is dominant in problems involving defects. As an illustration, we compute the differential cross section of scatterings of low-energy transverse elastic phonons by a superfluid vortex. The result is model-independent.
\end{abstract}

PACS numbers: $67.80 .-s, 11.10 . E f$ 
Introduction. - The possibility of superfluid behavior in solids was considered by Andreev and Lifshitz (AL) in a seminal paper of 1969 [1]. They proposed that point defects in a ${ }^{4} \mathrm{He}$ crystal may become quantum at low temperatures and form a Bose condensate. The possibility of superfluid solids ("supersolids") was also conjectured in Refs. [2, 3]. For a long time, the experimental search for a supersolid phase of helium was unsuccessful. Recently, however, Kim and Chan claimed that superfluid behavior may have been observed in solid ${ }^{4} \mathrm{He}[4,5]$. This has stimulated renewed interest in the supersolid phase. The superfluid fraction was found in Ref. [5] to be of order $10^{-2}$, considerably larger than what was theoretically expected $\left(\lesssim 10^{-4}[3]\right)$.

In the following, we assume that the supersolid phase does indeed occur. We shall not discuss the microscopic origin of such a behavior. Rather, we concentrate our attention on the theoretical description of the low-energy dynamics of the supersolid phase. One can expect that, regardless of the details of the microscopic mechanism underlying the supersolid state, its low-energy dynamics is simple and universal, and is dictated by symmetry principles alone. This is because the low-energy degrees of freedom of supersolids are the NambuGoldstone bosons arising from spontaneous breaking of translational symmetry and the U(1) symmetry generated by the conserved particle number. In fact, Andreev and Lifshitz have constructed the hydrodynamic equations describing a supersolid (in the limit of small strain), based on conservation laws and Galilean invariance only.

In this Letter, we restrict ourselves to zero temperature and derive an alternative description of supersolids based on a low-energy effective Lagrangian [6]. Such a description is possible since dissipative effects disappear at $T=0$. The effective Lagrangian description holds several advantages over the one based on the hydrodynamic equations. As it is generally true, the Lagrangian formulation enables straightforward application of field-theoretical techniques such as Feynman diagrams. In our particular case, the Lagrangian also elucidates the appearance of a certain "topological" interaction term, which is important in processes involving defects.

The form of the effective Lagrangian is constrained by various symmetries, among which an important role is played by the Galilean invariance. For liquid superfluids, the most general Galilean-invariant effective Lagrangian was constructed by Greiter, Wilczek, and Witten [7]. For supersolids the possible structures appearing in the Lagrangian is richer than in supersolid ${ }^{4} \mathrm{He}$, but are still rather restrictive. 
Degrees of freedom.- The fields appearing in the effective Lagrangian are the four Goldstone bosons which appear due to the spontaneous breaking of the $\mathrm{U}(1)$ particle number symmetry and the translational symmetry along three spatial directions. One of these fields is therefore the phase of the superfluid condensate, $\theta$; under the action of the particle number, it transforms as $\theta \rightarrow \theta+\alpha$.

The three remaining fields are translation-breaking scalars $X^{a}, a=1,2,3$, which can be introduced as follows [8]. Imagine a system of coordinates $X^{a}, a=1,2,3$ which is frozen in the body of the solid. (The choice of the system is completely arbitrary, but for simplicity one can choose it to coincide with the coordinates in our reference frame $x^{i}, i=1,2,3$ if the solid is at equilibrium at some arbitrarily chosen external pressure $P_{0}$.) When the solid moves, this system of coordinates also moves, so if one follows one particular material point in the solid, its coordinates in the $X$ system remain constant. In general, the coordinate system $X$ is curved. The time history of the solid is completely characterized by three functions $X^{a}(t, \mathbf{x})$, which give the coordinates, in the comoving frame, of the material point that is located at the position $\mathbf{x}$ at time $t . X^{a}(t, \mathbf{x})$ are the fields that enter the effective theory together with the $\mathrm{U}(1)$ phase $\theta$.

One can expand the fields around the ground state

$$
\theta=\mu_{0} t-\varphi, \quad X^{a}=x^{a}-u^{a},
$$

where $\varphi$ and $u^{a}$ fluctuate around zero. Here $\mu_{0}$ is the chemical potential at pressure $P_{0} ; u^{a}$ is the usual displacement vector. The superfluid velocity and the strain of the crystal are related to the first spatial derivatives of $\theta$ and $X^{a}$ :

$$
\mathbf{v}_{s}=\frac{1}{m} \boldsymbol{\nabla} \varphi, \quad \partial_{i} X^{a}=\delta_{i a}-\partial_{i} u^{a}
$$

(in most of this Letter, $\hbar=1$ ).

The density of lattice sites is a constant in $X$ space. We denote this constant by $n_{0}$. In the $x$ space, the density of lattice sites is

$$
n_{0} \operatorname{det}\left|\partial_{i} X^{a}\right|=\frac{n_{0}}{6} \epsilon_{i j k} \epsilon_{a b c} \partial_{i} X^{a} \partial_{j} X^{b} \partial_{k} X^{c} .
$$

For an ordinary crystal, nonsuperfluid and without defects, this coincides with the particle number density.

Derivative expansion. - The effective Lagrangian should be invariant under the U(1) particle number symmetry, $\theta \rightarrow \theta+\alpha$, and coordinate shift in the frozen frame, $X^{a} \rightarrow X^{a}+\alpha^{a}$. 
Thus the Lagrangian contains only time and coordinate derivatives of $\theta$ and $X^{a}$, but not $\theta$ or $X^{a}$ by themselves.

In order to discuss the low-energy regime, we follow the standard effective field theory philosophy and perform a derivative expansion of the effective Lagrangian. Because $\theta$ and $X^{a}$ do not appear without derivatives, there are two different ways to perform the derivative expansion. One possibility is to assume that $\theta$ and $X^{a}$ are slowly varying functions of space and time. Alternatively, one can assume that the first (temporal and spatial) derivatives of $\theta$ and $X^{a}$ vary slowly. Clearly, the second alternative is more general, since it allows for the first derivatives of $\theta$ and $X^{a}$ to be large. In particular, the superfluid velocity and the strain of the crystal do not have to be small. In our subsequent discussion, we will therefore assume that $\dot{\theta}, \partial_{i} \theta, \dot{X}^{a}$ and $\partial_{i} X^{a}$ may be not small, but vary slowly in space and time over distances large compared to all microscopic length scales, such as the superfluid healing length.

Keeping only leading-order terms in the derivative expansion, the Lagrangian is a function of the first derivatives of fields,

$$
\mathcal{L}=\mathcal{L}\left(\dot{\theta}, \partial_{i} \theta, \dot{X}^{a}, \partial_{i} X^{a}\right)
$$

This Lagrangian, in general, contains terms to all orders of fields. In each term in the series expansion over fields $\theta$ and $X^{a}$, one keeps the lowest possible number of derivatives equal to the number of fields.

Rotational invariance.- The effective Lagrangian should be invariant under spatial rotations. The fields $X^{a}$, despite being a three-component field, transform under spatial rotations like scalar fields. This is because $X^{a}$ are the coordinates of the internal system frozen in the solid body, which are not rotated with the axes of spatial coordinates $x^{i}$. Therefore the Lagrangian (4) is a function of the following rotationally invariant combinations of arguments,

$$
\mathcal{L}=\mathcal{L}\left(\dot{\theta}, \dot{X}^{a},\left(\partial_{i} \theta\right)^{2}, \partial_{i} \theta \partial_{i} X^{a}, u^{a b}\right)
$$

where we introduce the notation

$$
u^{a b}=\partial_{i} X^{a} \partial_{i} X^{b}=\delta^{a b}-\partial_{a} u^{b}-\partial_{b} u^{a}+\partial_{i} u^{a} \partial_{i} u^{b} .
$$

It can be shown that any rotationally invariant function of first derivatives of $\theta$ and $X^{a}$ can 
be written as a function of the parameters staying in Eq. (5). In particular,

$$
\begin{aligned}
& \epsilon_{i j k} \partial_{i} X^{a} \partial_{j} X^{b} \partial_{k} X^{c}=\epsilon^{a b c} \sqrt{\operatorname{det} u} \\
& \epsilon_{i j k} \partial_{i} \theta \partial_{j} X^{a} \partial_{k} X^{b}=\epsilon^{a b c} \sqrt{\operatorname{det} u} u_{c d}^{-1} \partial_{i} \theta \partial_{i} X^{d},
\end{aligned}
$$

where det $u$ is the determinant of the $3 \times 3$ matrix $u^{a b}$, and $u_{a b}^{-1}$ is the inverse matrix of $u$ : $u_{a b}^{-1} u_{b c}=\delta_{a c}$.

Galilean invariance.-Further constraints on the effective Lagrangian follow from Galilean invariance. In a nonrelativistic theory where all particles have the same mass, the momentum density is proportional to the particle number flux,

$$
T^{0 i}=m j^{i}
$$

where $m$ is the mass of the ${ }^{4} \mathrm{He}$ atom. The momentum density and the particle number flux are found from the Lagrangian by using Noether's theorem,

$$
T^{0 i}=-\frac{\partial \mathcal{L}}{\partial \dot{\theta}} \partial_{i} \theta-\frac{\partial \mathcal{L}}{\partial \dot{X}^{a}} \partial_{i} X^{a}, \quad j^{i}=\frac{\partial \mathcal{L}}{\partial\left(\partial_{i} \theta\right)} .
$$

The most general form of the Lagrangian which is consistent with rotational [Eq. (5)] and Galilean invariance [Eq. (9)] is

$$
\mathcal{L}=\mathcal{L}\left(\mu, w^{a}, u^{a b}\right)
$$

Here $u^{a b}$ was defined in Eq. (6), and $\mu$ and $w^{a}$ are

$$
\mu=\dot{\theta}-\frac{\left(\partial_{i} \theta\right)^{2}}{2 m}, \quad w^{a}=-\dot{X}^{a}+\frac{1}{m} \partial_{i} \theta \partial_{i} X^{a} .
$$

The variable $\mu$ appears in the Lagrangian treatment of superfluids [7]: the crystal structure is absent there and the Lagrangian is a function of $\mu$ alone. Physically, $\mu$ is the local chemical potential as measured in the frame moving with the superfluid velocity. The meaning of $w^{a}$ can be made clear by expanding it,

$$
\mathbf{w}=\dot{\mathbf{u}}+\left(\mathbf{v}_{s} \cdot \nabla\right) \mathbf{u}-\mathbf{v}_{s}
$$

At the linearized level $w^{a}$ is the difference between the velocity of motion of the crystal lattice and the superfluid velocity.

Connection to the AL hydrodynamic theory. - The zero-temperature AL hydrodynamic equations can be derived from the Lagrangian (11) as the equations of conservation of particle 
number, energy, and momentum, written using a particular set of variables. We introduce the total density $\rho$, the superfluid velocity $\mathbf{v}_{s}$, and the vector $\mathbf{v}_{n}$ which corresponds to the normal velocity in the AL theory,

$$
\rho=m \frac{\partial \mathcal{L}}{\partial \mu}, \quad v_{s i}=\frac{1}{m} \partial_{i} \varphi, \quad v_{n i}=\frac{\partial x^{i}}{\partial X^{a}} \dot{u}^{a} .
$$

Actually $\mathbf{v}_{n}$ is the velocity of the crystal lattice; at the linearized level $\mathbf{v}_{n}=\dot{\mathbf{u}}$. At zero temperature we do not have a normal component distinct from the crystal lattice. Furthermore, we introduce the momentum density $\mathbf{p}$ and the energy density $\varepsilon$ in the frame $\mathbf{v}_{s}=0$,

$$
p_{i}=\frac{\partial \mathcal{L}}{\partial w^{a}} \partial_{i} X^{a}, \quad \varepsilon=\mu \frac{\partial \mathcal{L}}{\partial \mu}+w^{a} \frac{\partial \mathcal{L}}{\partial w^{a}}-\mathcal{L},
$$

and an auxiliary tensor $\lambda_{i a}$,

$$
\lambda_{i a}=2 \frac{\partial \mathcal{L}}{\partial u^{a b}} \partial_{i} X^{b}+\left(v_{n i}-v_{s i}\right) \frac{\partial \mathcal{L}}{\partial w^{a}} .
$$

After some algebra, we find that the momentum density can be written as

$$
T^{0 i}=m j^{i}=\rho v_{s i}+p_{i}
$$

the energy density and the energy flux as ${ }^{1}$

$$
\begin{aligned}
& T^{00}=\frac{\rho v_{s}^{2}}{2}+\mathbf{p} \cdot \mathbf{v}_{s}+\varepsilon \\
& T^{i 0}=\left(\mu+\frac{m v_{s}^{2}}{2}\right) j^{i}+v_{n i}\left(\mathbf{v}_{n} \cdot \mathbf{p}\right)-\lambda_{i a} \dot{u}^{a},
\end{aligned}
$$

and the differential of $\varepsilon$ as

$$
d \varepsilon=\frac{\mu}{m} d \rho+\left(\mathbf{v}_{n}-\mathbf{v}_{s}\right) \cdot d \mathbf{p}+\lambda_{i a} d\left(\partial_{i} u^{a}\right)
$$

Equations (17)-(20) are identical to the corresponding AL equations at $T=0$. The stress tensor can be transformed into the form

$$
T^{i k}=\rho v_{s i} v_{s k}+v_{s k} p_{i}+v_{n i} p_{k}+\delta_{i k}\left[\mu \frac{\rho}{m}+\left(\mathbf{v}_{n}-\mathbf{v}_{s}\right) \cdot \mathbf{p}-\varepsilon\right]-\lambda_{i k}+\lambda_{i a} \partial_{k} u^{a},
$$

which almost coincides with the corresponding AL expression. The only difference is the last term on the right hand side of Eq. (21), which is nonlinear in strain and was neglected

\footnotetext{
${ }^{1}$ Note that $T^{0 i} \neq T^{i 0}$, since in our nonrelativistic theory energy does not include rest mass. Our normalization of the chemical potential differs from that of AL by a factor of $m$.
} 
in Ref. [1]. Moreover, from the definition of $\lambda_{i a}$, Eq. (16). one can derive the following relation:

$$
\lambda_{i k}-\lambda_{k i}-\lambda_{i a} \partial_{k} u^{a}+\lambda_{k a} \partial_{i} u^{a}=\left(v_{n i}-v_{s i}\right) p_{k}-\left(v_{n k}-v_{s k}\right) p_{i},
$$

which coincides, up to the two terms nonlinear in strain on the left hand side, to an equation postulated in Ref. [1] for $T^{i k}$ to be a symmetric tensor.

As one can see, the effective Lagrangian provides an extremely compact encoding of the hydrodynamic equations. Terms nonlinear in strain which were omitted in the AL theory are fully kept in the Lagrangian. Moreover, for quantum problems (such as scatterings of phonons) it is easier to work with the Lagrangian than with the field equations. We now show that the Lagrangian contains a special topological term that is important for scattering off defects. For this end, we first discuss the nonsuperfluid limit of the Lagrangian (11).

The nonsuperfluid limit-The superfluid fraction $\rho_{s} / \rho$ of solid helium-4, if nonzero, is much smaller than one: experiments $[4,5]$ indicate a value of order $10^{-2}$, while theoretical arguments [3] suggest $\rho_{s} / \rho \lesssim 10^{-4}$. We shall, therefore, concentrate on the limit $\rho_{s} \ll \rho$. To start, let us discuss the limit of vanishing superfluid density.

One expects that the nonsuperfluid crystalline state is realized as some particular limit of the supersolid state, where $\theta$ decouples from the dynamics of $X^{a}$ fields. This might seem nontrivial, since in Eq. (11) the time derivative of $X^{a}$ enters the Lagrangian in the combination $w^{a}$ which involves $\theta$ [Eq. (12)]. However, it is possible to achieve such a decoupling. Consider the following Lagrangian:

$$
\mathcal{L}=\rho_{0} \sqrt{\operatorname{det} u}\left(\frac{1}{2} u_{a b}^{-1} w^{a} w^{b}+\frac{\mu}{m}\right)-V\left(u^{a b}\right),
$$

where $\rho_{0}$ is some constant with the dimension of mass density, $\mu$ and $w$ are defined in Eq. (12), and $V$ is an arbitrary function of the strain $u^{a b}$ consistent with lattice symmetry. Using the identities (7) and (8), this Lagrangian can be transformed into the form

$$
\mathcal{L}=\frac{\rho_{0}}{2} \sqrt{\operatorname{det} u} u_{a b}^{-1} \dot{X}^{a} \dot{X}^{b}-V\left(u^{a b}\right)+\frac{\rho_{0}}{6 m} \epsilon^{\mu \nu \lambda \rho} \epsilon_{a b c} \partial_{\mu} \theta \partial_{\nu} X^{a} \partial_{\lambda} X^{b} \partial_{\rho} X^{c}
$$

The Greek indices in the last term are spacetime indices which run over $t, x, y, z ; \epsilon^{\mu \nu \lambda \rho}$ is the completely antisymmetric tensor defined so that $\epsilon^{0123}=1$. The phase $\theta$ appears only in the last term of the Lagrangian, which is a full derivative; thus $\theta$ decouples from the dynamics. 
The last term, which will be called the "topological term," still plays a useful role: the particle number current computed from (24) by using Noether's theorem arises completely from this term,

$$
j^{\mu}=\frac{\rho_{0}}{6 m} \epsilon^{\mu \nu \lambda \rho} \epsilon_{a b c} \partial_{\nu} X^{a} \partial_{\lambda} X^{b} \partial_{\rho} X^{c}
$$

from which we see that $\rho_{0}$ is the total mass density in equilibrium where $X^{a}=x^{a}$. The current (25) is trivially conserved. Note that the first term in Eq. (24) can be written as $m j^{i} j^{i} /\left(2 j^{0}\right)$, which is what one expects for the kinetic energy from Galilean invariance. This fact shows that the construction (23) is unique.

Now if one allows the superfluid fraction $\rho_{s}$ to be nonzero and small, then there are additional terms proportional to $\rho_{s}$ added to the Lagrangian, which makes $\theta$ a dynamical field. The topological term continues to be present in the Lagrangian with a coefficient which differs only slightly from $\rho /(6 \mathrm{~m})$. This term is responsible for low-energy scattering of elastic waves by a superfluid vortex, as we shall see.

Scattering of elastic waves by a superfluid vortex.-Let us now use the effective Lagrangian to compute the scattering cross section of elastic waves by a superfluid vortex. In the presence of a vortex $\varphi$ is a multivalued function, and $\mathbf{v}_{s} \sim \nabla \varphi$ is singular at the vortex core. Due to the multivalued nature of $\theta$, the topological term is no longer integrated by part to zero. Integrating by part, this term can be written as

$$
\mathcal{L}_{\text {top }}=-\frac{\rho}{6 m} \epsilon^{\mu \nu \lambda \rho} \epsilon^{a b c} \partial_{\mu} \partial_{\nu} \varphi X^{a} \partial_{\lambda} X^{b} \partial_{\rho} X^{c}
$$

Expanded over small perturbations, this expression contains a term proportional to $\mathbf{u} \cdot \dot{\mathbf{v}}_{s}$, which was identified in Ref. [9] in the context of an Abrikosov vortex in a crystal. For definiteness, consider a vortex located at $x=y=0$ and stretched along the $z$ direction. The field of the vortex has

$$
\partial_{x} \partial_{y} \varphi-\partial_{y} \partial_{x} \varphi=2 \pi \delta(x) \delta(y)
$$

The topological term is localized on the vortex core and has the form

$$
\mathcal{L}=-\pi \frac{\rho}{m} \delta(x) \delta(y)\left(u_{x} \dot{u}_{y}-u_{y} \dot{u}_{x}\right)+O\left(u^{3}\right)
$$

Note that the leading term in this Lagrangian contains two powers of $u$ but only one derivative, so it cannot be canceled by an unknown interaction of the vortex core with the elastic 


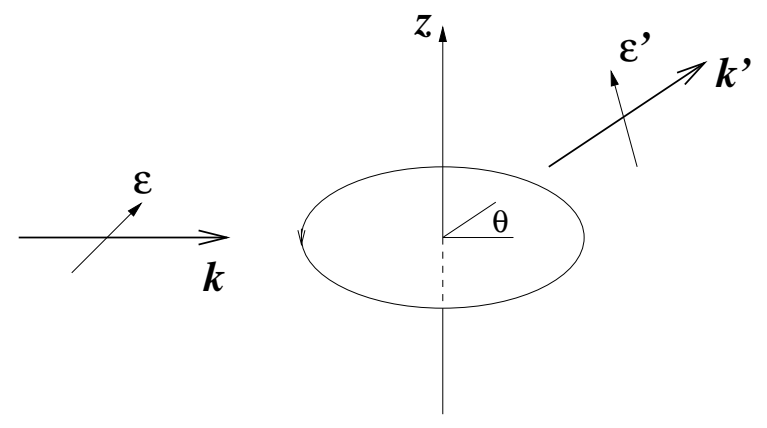

FIG. 1: The scattering of transverse elastic phonons by a superfluid vortex.

waves. Moreover, other terms that couple $\theta$ and $X^{a}$ are expected to be proportional to $\rho_{s}$ and are negligible.

The process that will be considered has the following kinematics (Fig. 1). A transverse phonon with momentum $\hbar \mathbf{k}$ and linear polarization $\boldsymbol{\epsilon}$ falls perpendicularly onto a vortex. We are interested in the probability of its scattering into the state with momentum $\hbar \mathbf{k}^{\prime}$ and polarization $\boldsymbol{\epsilon}^{\prime}$. For simplicity, we assume that both $\boldsymbol{\epsilon}$ and $\boldsymbol{\epsilon}^{\prime}$ lie in the plane perpendicular to the vortex and that the solid is isotropic. The matrix element of the process is

$$
M=\frac{\pi}{m}\left(\boldsymbol{\epsilon} \times \boldsymbol{\epsilon}^{\prime}\right) \cdot \hat{\mathbf{z}},
$$

from which we find the differential cross section per unit vortex length,

$$
\frac{\partial^{2} \sigma}{\partial \theta \partial l}=\frac{\pi}{2} \frac{\hbar^{2}}{m^{2} v_{\perp}^{2}} k \sin ^{2} \theta,
$$

where $v_{\perp}$ is the speed of transverse elastic waves. This result is a model-independent prediction of the effective Lagrangian approach, valid at small $k$ and small superfluid fraction. Qualitatively, the differential cross section has a linear dependence on $k$ and is maximum when the scattering angle $\theta$ is $90^{\circ}$.

Conclusion.-We have found the most general effective Lagrangian describing the lowenergy dynamics of supersolids. We show that, in the limit of small superfluid density, the Lagrangian contains a topological term which has a fixed coefficient. From this term we computed the cross section of scattering of transverse phonons off a superfluid vortex. If the supersolid state is realized in ${ }^{4} \mathrm{He}$, this prediction is, in principle, verifiable.

The formalism used in this paper can be extended to relativistic systems, e.g., for describing the crystalline superfluid phases of quark matter [10]. Instead of Galilean invariance, one requires relativistic invariance of a theory of four Goldstone bosons $\theta$ and $X^{a}$. Such a 
theory, when coupled to gravity, gives rise to a gravitational analog of the Anderson-Higgs mechanism. Indeed, theories of this type have been proposed recently as an infrared modification of gravity that gives the graviton Lorentz-breaking mass terms [11, 12]. From this point of view, the modification of gravity considered in Refs. [11, 12] can be interpreted as an effect coming from a supersolid dark matter sector.

The author thanks A. Andreev, J. Erlich, P. Kovtun, M. A. Stephanov, and D. J. Thouless for discussions. This work was supported by DOE grant DE-FG02-00ER41132 and the Alfred P. Sloan Foundation.

[1] A. F. Andreev and I. M. Lifshitz, Sov. Phys. JETP 29, 1107 (1969).

[2] G. V. Chester, Phys. Rev. A 2, 256 (1970).

[3] A. J. Legett, Phys. Rev. Lett. 25, 1543 (1970).

[4] E. Kim and M.H.W. Chan, Nature 427, 225 (2004).

[5] E. Kim and M.H.W. Chan, Science 305, 1941 (2004).

[6] S. Weinberg, Physica (Amsterdam) 96A, 327 (1979).

[7] M. Greiter, F. Wilczek, and E. Witten, Mod. Phys. Lett. B 3, 903 (1989).

[8] H. Leutwyler, Helv. Phys. Acta 70, 275 (1997).

[9] E. M. Chudnovsky and A. B. Kuklov, Phys. Rev. Lett. 91, 067004 (2003).

[10] M. Alford, J. A. Bowers, and K. Rajagopal, Phys. Rev. D 63, 074016 (2001).

[11] V. Rubakov, hep-th/0407104.

[12] S. L. Dubovsky, J. High Energy Phys. 10 (2004) 076. 